\title{
4 Algorithmen und Diagramme
}

Es ist evident, dass heute selten wissenschaftliche Diagramme ohne Hilfe des Computers erstellt werden. Selbst wenn sie einen „handstiftlichen“ Ursprung haben, wie etwa der Kreis in Abbildung 1 ganz zu Beginn dieses Buches, werden sie meist digitalisiert und dann in ihrer digitalen Form weiterverarbeitet. Der Fokus dieses Kapitels liegt jedoch auf Diagrammen, die aufgrund empirischer Daten über ein Set von Anweisungen, also algorithmisch, automatisch erstellt werden. Dies ist bereits der Fall, wenn eine Liste von Wertpaaren, z. B. Frequenzen eines Wortes in einem Korpus in verschiedenen Jahren, also eine Zeitreihe, mittels geeigneter Software mit einem Klick in ein Balkendiagramm umgeformt wird. Ein anderes Beispiel ist die automatische Platzierung von Datensätzen zu Aussprachevarianten eines Lexems an bestimmten Erhebungsorten auf einer Karte, was zu einer Dialektkarte führt.

Warum sollen so erstellte Diagramme einen Sonderstatus innehaben? Ist der einzige Unterschied zum manuell erstellten Diagramm nicht bloß der Aspekt der Quantität? Das maschinell erstellte Balkendiagramm wird exakter sein als das von Hand gezeichnete, selbst wenn mit größter Sorgfalt, d. h. auf Millimeterpapier mit Lineal und sorgsam gespitztem Bleistift gezeichnet wird. Und es wird schneller erstellt sein, besonders dann, wenn nicht nur eine Handvoll Datenpaare, sondern Hunderte, Tausende oder Millionen Datensätze zu visualisieren sind.

Das ist fraglos richtig, doch sind es zwei weitere Aspekte, die eine genauere Betrachtung algorithmisch erstellter Diagramme rechtfertigen:

Schon seit über zehn Jahren wird im Kontext der wissenschaftlichen Visualisierungen dem Paradigma der „Visual Analytics“ besondere Aufmerksamkeit und damit verbundene Hoffnung zuteil. Methoden der Visual Analytics sollen dort zum Einsatz kommen, wo die zu analysierenden Daten nicht mehr einfach überblickbar sind und deswegen weiter transformiert werden, so dass interaktive visuelle Interfaces die Analyse erst ermöglichen:

Visual analytics is the science of analytical reasoning facilitated by interactive visual interfaces. People use visual analytical tools and techniques to synthesize information and derive insights from massive, dynamic, ambiguous, and often conflicting data; detect the expected and discover the unexpected; provide timely, defensible, and understandable assessments; and communicate assessment effectively for action. (Thomas/Cook 2005, 4)

Das Zitat nennt die relevanten Merkmale: Große Datenmengen, effiziente Analyse, neue Erkenntnisse. Im Zusammenhang mit größer werdenden Datengrundlagen auch in den Geistes- und Sozialwissenschaften und insbesondere auch in der Linguistik, etwa in der Form von Textkorpora, wird solchen Methoden auch da großen Nutzen zugesprochen. Gerade unter dem Label „Digital Humani- 
ties“ erfreut sich Visual Analytics einiger Beliebtheit. Sie sollen, Franco Morettis Programm des „Distant Readings“ (Moretti 2009) folgend, eine „multi-faceted view of the textual data“ (Jänicke et al. 2016) ermöglichen. Für die Geschichtswissenschaft konstatiert Richard White:

\footnotetext{
visualization and spatial history are not about producing illustrations or maps to communicate things that you have discovered by other means. It is a means of doing research; it generates questions that might otherwise go unasked, it reveals historical relations that might otherwise go unnoticed, and it undermines, or substantiates, stories upon which we build our own versions of the past. (White 2010, Hervorh. im Original)
}

Eine diagrammatische Aufbereitung der Daten soll also Zugriffe auf Daten erlauben, die bisher nicht möglich waren, wobei diese neuen Zugriffe nicht nur durch die Visualisierung selbst, sondern eben durch die algorithmische Visualisierung möglich sein soll. Wenn es also nicht das Diagramm an und für sich ist, was die neuen Erkenntnisse ermöglicht (und was nach den Ausführungen in Kapitel 2 zur Diagrammatik auch nicht überraschend wäre), muss das Algorithmische genauer betrachtet werden - allerdings unter diagrammatischen Gesichtspunkten.

Diese Reflexionen führen jedoch automatisch zum zweiten Aspekt, warum sich ein genauer Blick auf algorithmische Visualisierungen lohnt: Während in den Visual Analytics die Visualisierung dort beginnt, wo Daten zu grafischen Formen ,gemappt ' werden, also eine Karte, ein Netzwerk o. ä. maschinell gezeichnet wird, ist aus diagrammatischer Sicht fragwürdig, ob diese Visualisierung nicht bloß ein kleiner Teilaspekt dessen ist, was das Diagramm ausmacht und viele Schritte davor nicht bereits viele wichtige diagrammatische Operationen im Spiel sind. Daher wird an dieser Stelle die Frage zentral: Wie verhält sich der Algorithmus zum Diagramm?

Diese Fragen werden im Folgenden angegangen, wobei zunächst die Verdatung von Sprache als Grundlage für alle weiteren Operationen diskutiert werden soll.

\subsection{Verdatung von Sprache}

Der Computer ist nicht nur, wie häufig angenommen wird, eine Rechenmaschine, sondern ganz grundlegend auch ein textverarbeitendes System. Der Text ist Code, der in Schaltzustände übersetzt wird und das Ensemble der Schaltzustände steht dann für Werte und Operationen, die wieder zu neuen Schaltzuständen führen, die so dargestellt werden, dass sie auch menschlich interpretierbar sind, d.h. als Text. Wichtig ist zunächst jedoch der Schritt der Codierung, der nötig ist, um Daten und Operationen als Schaltzustände, also digital, zu repräsentieren. 
Wenn wir nun an die Digitalisierung von sprachlichen Daten denken, ist „Digitalisierung “ ein sehr ungenauer Begriff für diesen Vorgang, was selbstredend an den verschiedenen Erscheinungsformen von Sprache liegt: Erscheinungsformen sind z. B. gesprochene oder geschriebene Sprache, von sprachlichen Äußerungen abstrahierte Eigenschaften wie Phoneme, Morpheme, Wörter, syntaktische Strukturen etc. Man kann einen Computer mit einer Tabelle von Werten, die von Sprachgebrauch abgeleitet sind, füttern oder aber sozusagen mit so genannten Rohdaten, also sprachlichen Äußerungen. Diese können, müssen aber nicht zwingend, zunächst in ein digitales Format überführt werden, etwa indem ein Text digital codiert oder eine Lautäußerung in ein digitales Sprachsignal überführt werden. Dies macht die ursprünglichen Informationen für den Computer erreichbar, da sie so codiert und elektronisch repräsentiert sind, um zum Gegenstand von Anweisungen (Programmcode) werden zu können.

Dies alleine macht den Reiz von Datenanalyse jedoch noch nicht aus, da die sprachlichen Äußerungen jetzt zwar digital vorliegen, jedoch noch keine Daten sind; sie müssen noch verdatet werden (Bubenhofer/Scharloth 2015). Mit der Verdatung werden weitere Abstraktionsebenen über die digital codierten Informationen gelegt, wobei diese mehr oder weniger abstrakt sein können. Bei digital codierter geschriebener Sprache gehören linguistische Informationen dazu, etwa die Information, wo ein Text beginnt und endet, wo Wort- und Satzgrenzen liegen und andere basale Aspekte, die z. B. in der Korpuslinguistik als grundlegende korpuslinguistische Verarbeitungsschritte angesehen werden (Lemnitzer/Zinsmeister 2006; Perkuhn et al. 2012). Darauf bauen abstraktere Ebenen auf, die häufig sprachliche Einheiten auszählen und Werte aggregieren. Dazu ein Beispiel:

$\mathrm{Zu}$ allen Wörtern (Types) in einem Textkorpus werden Kollokationsprofile erstellt, also ausgezählt, welche anderen Wörter typischerweise in ihrer Umgebung auftreten. Um dies zu ermöglichen, wird eine Matrix erstellt, die für jedes Wort die Häufigkeiten nennt, mit denen es zusammen mit anderen Wörtern auftritt. Daraus ergibt sich für jedes Wort eine Reihe von Zahlen, die für die Frequenzen der kollokierenden Wörter stehen. Diese Zahlenreihe ist in gewisser Weise eine Art Fingerabdruck des jeweiligen Wortes - es handelt sich um einen Zahlenvektor:

Tab. 3: Zahlenvektor

\begin{tabular}{lllllllllll}
\hline & weiß & Tür & Gefühl & essen & Garten & fahren & Zug & grün & Das & $\cdot$ \\
\hline Haus & 1 & 1 & 0 & 0 & 1 & 0 & 0 & 0 & 1 & 1 \\
Bahnhof & 0 & 1 & 0 & 0 & 0 & 1 & 1 & 0 & 0 & 1 \\
$\ldots$ & & & & & & & & & &
\end{tabular}


In diesem erfundenen Beispiel ist das Kollokationsverhalten von „Haus“ durch die Zahlenreihe $\{1,1,0,0,1,0,0,0,1,1\}$ bestimmt. 1 bedeutet, dass das Wort „Haus“ mit dem als Spaltentitel genannten Wort zusammen auftritt, 0, dass es nicht damit auftritt. „Haus“ - so nehmen wir in diesem erfundenen Beispiel an - tritt also zusammen mit „weiß“, „Tür“, „Garten“, „Das“ und dem Punkt auf, jedoch nicht mit den anderen Wörtern. Dies drückt der Zahlenvektor aus. Das Kollokationsverhalten von „Bahnhof“ ist mit $\{0,1,0,0,0,1,1,0,0,1\}$ bestimmt, es tritt zusammen mit „Tür“, „fahren“, „Zug“ und dem Punkt auf.

So kann die Semantik - wenn man das Kollokationsverhalten in einem geeigneten Korpus als Operationalisierung von Semantik im Sinne einer Gebrauchsbedeutung hinnehmen möchte - mathematisch als Zahlencode, als Vektor, ausgedrückt werden.

Um zu den Vektoren zu gelangen, waren eine Reihe von diagrammatischen Operationen nötig, mit denen die Daten umformatiert werden. Auf das obige Beispiel bezogen, wären dies die Folgenden: In einem Textkorpus werden alle Belege für „Haus“ (bzw. für „Bahnhof“) gesucht und aufgelistet. Hier ein Beispiel für eine Suche nach „Haus“ in einem Korpus:

Tab. 4: KWiC-Ansicht

\begin{tabular}{rll}
\hline Das Weiße & Haus & entschied sich gegen die Reform. \\
Das verwunschene & Haus & besaß weder Tür noch Fenster. \\
Nur Spießer träumen von & Hauses & $\cdot$ \\
Vor dem & Haus & und Garten! \\
Der Präsident geht ins Weiße & Haus & um zu twittern. \\
\hline
\end{tabular}

Diese typische „Key Word in Context“ (KWiC)-Ansicht zeigt auf übersichtliche Weise die Kontexte des Lemmas „Haus“. Grundlage dieser Ansicht ist ein permutiertes Register, bei dem ausgehend von einem Korpus jedes Wort (Token) einmal als Index für seinen Kontext dient. Das bedeutet, dass der Reihe nach jedes Token im Korpus als Index aufgeführt wird, das auf seinen ursprünglichen Kontext im Text verweist: 
Tab. 5: Index (Korpus: Das verwunschene Haus besaß weder Türen noch Fenster.)

\begin{tabular}{ll}
\hline Index (mit Lemma) & Kontext \\
\hline Das (der|die|das) & Das verwunschene Haus besaß \\
verwunschene (verwunschen) & Das verwunschene Haus besaß weder \\
Haus (Haus) & Das verwunschene Haus besaß weder Türen \\
besaß (besitzen) & Das verwunschene Haus besaß weder Türen noch \\
weder (weder) & verwunschene Haus besaß weder Türen noch Fenster \\
Türen (Tür) & Haus besaß weder Türen noch Fenster . \\
noch (noch) & besaß weder Türen noch Fenster . \\
Fenster (Fenster) & weder Türen noch Fenster . \\
. & Türen noch Fenster . \\
\hline
\end{tabular}

Um eine KWiC-Liste zu „Haus“ daraus zu generieren, reicht es, die Zeile mit dem Index „Haus“ abzurufen, wofür der permutierte Index idealerweise alphabetisch sortiert vorliegt:

Tab. 6: Index alphabetisch

\begin{tabular}{ll}
\hline Index (mit Lemma) & Kontext \\
\hline & Türen noch Fenster . \\
besaß (besitzen) & Das verwunschene Haus besaß weder Türen noch \\
Das (der|die|das) & Das verwunschene Haus besaß \\
Fenster (Fenster) & weder Türen noch Fenster . \\
Haus (Haus) & Das verwunschene Haus besaß weder Türen \\
noch (noch) & besaß weder Türen noch Fenster . \\
Türen (Tür) & Haus besaß weder Türen noch Fenster . \\
verwunschene (verwunschen) & Das verwunschene Haus besaß weder \\
weder (weder) & verwunschene Haus besaß weder Türen noch Fenster \\
\hline
\end{tabular}

Wäre das Korpus größer, ergäben sich für „Haus“ entsprechend mehr Zeilen, die in die KWiC-Liste übernommen werden könnten. 
Um nun die Kollokationen von „Haus“ zu finden, muss die KWiC-Liste, die aus der Suche nach „Haus“ entstanden ist (Tabelle 4) wiederum umgeformt werden, etwa in folgender Art:

Tab. 7: Frequenzindex

\begin{tabular}{llllll}
\hline 4 & 1 & stand & 1 & besaß \\
2 & Tür & 1 & sommerlichen & 1 & an \\
2 & Garten & 1 & sich & 1 & geht \\
2 & Weiße & 1 & noch & 1 & um \\
2 & Das & 1 & lag & 1 & zu \\
1 & ! & 1 & gegen & 1 & Vor \\
1 & weder & 1 & entschied & 1 & Der \\
1 & von & 1 & ein & 1 & Spießer \\
1 & verwunschene & 1 & die & 1 & Präsident \\
1 & verträumter & 1 & des & 1 & Sie \\
1 & und & 1 & der & 1 & Reform \\
1 & träumen & 1 & dem & 1 & Nur \\
1 & twittern & 1 & ins & 1 & Hauses \\
& & & 1 & Fenster \\
\hline
\end{tabular}

Im Prinzip wird also für jedes in der KWiC-Liste vorkommende Wort ein neuer Index erstellt, sortiert, die Häufigkeit von Zeilen mit gleichem Index ermittelt und rückwärts sortiert nach Häufigkeit ausgegeben. Darin sind nun auch die fünf Wortformen enthalten (Punkt, „Tür“, „Garten“, „Weiße“, „Das“), die in der Grundform in der Zahlenreihe (Tabelle 3) vorkommen und dort mit 1 gekennzeichnet werden, wobei 1 dafür steht, dass die Kollokation mindestens zweimal vorkommt.

Das erfundene Beispiel stellt selbstverständlich keine sinnvolle Berechnung von Kollokationsprofilen dar; die Vektoren müssten um viele weitere Lemmata angereichert werden und sie könnten zudem gewichtet werden, so dass die Häufigkeit der Kollokation im Zahlenvektor berücksichtigt würde, indem dort nicht nur die Werte 1 und 0 erlaubt wären, sondern direkt die Häufigkeit angegeben würde.

Es geht mir aber an dieser Stelle darum zu zeigen, welche an und für sich sehr einfachen Transformationen von Daten (auflisten, sortieren, auszählen) dazu 
führen, die Daten in eine komplett andere Form zu bringen. Diagrammatische Formen der Darstellung spielen dabei offensichtlich eine entscheidende Rolle: Liste, Index, Matrix, Tabelle und weitere mehr.

Diese Umformungen sind im Prinzip keine komplexen Vorgänge. Würden sie jedoch manuell durchgeführt, wären sie bei großen Datenmengen äußerst langwierig. Dies dokumentieren nicht zuletzt Wörterbuchprojekte, wie etwa eindrücklich anekdotische Schilderungen zum „Thesaurus linguae latinae“ zeigen:

Die Zettel zu den einzelnen Autoren standen in den Kästen noch so, wie sie nach der Verzettelung abgeliefert worden waren, also nach Perikopen [Abschnitte, NB] und innerhalb dieser in der Wortfolge der Texte. Diese Anordnung mußte noch in die alphabetische umgeändert werden, durch die Spezialwörterverzeichnisse zu den einzelnen Autoren entstanden. Diese sehr wichtige Arbeit konnte nur im Büro und in fortlaufender Arbeitszeit ausgeführt werden. Dazu waren Feiertage nötig, und so blieben nicht wenige von uns in den letzten Universitätsferien, die der Thesaurus an der Georgia Augusta erlebte, nach Ferienbeginn noch in Göttingen und ordnete gemeinsam in vielstündigen Arbeitstagen den Wortschatz der Texte alphabetisch. Es war keine schwierige, sondern eine ganz vergnügliche Arbeit. Das brachte die Gemeinsamkeit und die Aussicht auf raschen und guten Verdienst (für die Stunde 1 M) mit sich. Wir fanden schnell heraus, wie wir uns in die Hände arbeiten konnten. Die großen Tische erleichterten das Auslegen nach Buchstaben und innerhalb dieser nach Schriften und Stellen. (Bögel 1996, 16-17)

Für den Thesaurus wurden seit 1893 eine Sammlung von etwa 10 Mio. Zetteln erstellt, um den Wortgebrauch aller aus der Antike bis ins 6. Jahrhundert erhaltenen literarischen und nichtliterarischen lateinischen Texte abbilden zu können. ${ }^{17}$ Die Beschreibungen des Arbeitsalltags von Bögel (1996) deuten an, wie für eine einfache Sortieraufgabe von Zetteln systematische, raumgreifende Operationen nötig waren, „große Tische“, um die Zettel in gewünschter Weise sortieren zu können.

Was heute mit digitalen Daten auf Knopfdruck geschieht, war damals eine groß angelegte, organisatorische Aufgabe des manuellen Zettelmanagements, die nur mit einer klaren hierarchischen Aufgabenverteilung überhaupt funktionieren konnte:

Zu den Kopisten und Lemmatisatoren kamen für die mechanische Material-Verzettelung die „Alphabetisatoren“, die Zettel-Ordner. Nun, für diese Arbeit brauchte man noch weniger Latein als fürs Abschreiben. (Oskar Hey im Nachwort zu Bögel 1996, 171)

17 Vgl. TTL $\rightarrow$ Über den TTL: http://www.thesaurus.badw-muenchen.de/ (letzter Zugriff: 22. September 2020). 
Der Computer sollte die Möglichkeiten dieser „diagrammatischen Operationen“ fundamental ändern, wie bereits die Ausführungen zu den grundlegenden computertechnischen Operationen zu Beginn des Abschnitts zeigten. Doch zunächst tritt zum Ende des Zweiten Weltkriegs Vannevar Bushs „Memex“ auf den Plan (vgl. Abbildung 31). Im viel zitierten Artikel „As We May Think“ skizziert Bush, Direktor des Büros für „Scientific Research and Development“ der US-Regierung, eine Maschine zur Verwaltung der eigenen Bibliothek, die er Memex nennt:

A memex is a device in which an individual stores all his books, records, and communcations, and which is mechanized so that it may be consulted with exceeding speed and flexibility. It is an enlarged intimate supplement to his memory. (Bush 1945, 121)

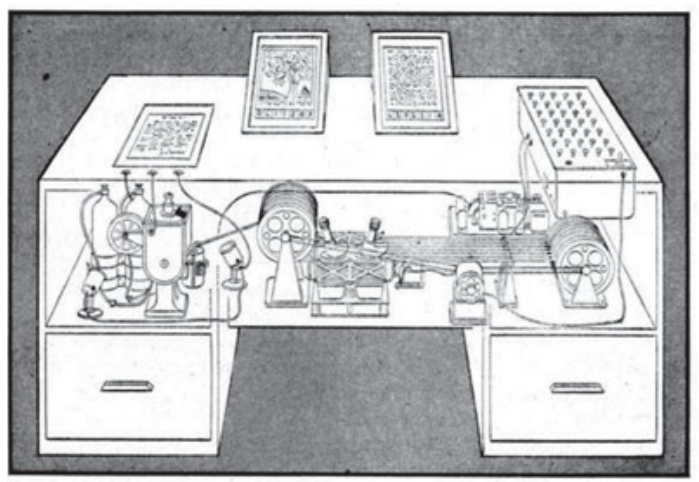

MEMEX in the form of a desk would instantly bring filcs and material on any subject to the operator's fingertips. Slanting translucent viewing screens magnify supermicrofilm filed by code numbers. At left is a mechanism which automatically photographs longhand notes, pictures and letters, then files them in the desk for future reference.

Abb. 31: Skizze von Bushs „Memex“ im Originaltext (Bush 1945, 123)

Grundlage des Memex sind die Möglichkeiten fotomechanischer Reproduktion mit Mikrofilmen. Jede zu speichernde Information wird als Mikrofilm abgelegt und über ein mechanisches System indiziert:

If the user wishes to consult a certain book, he taps its code on the keyboard and the title page of the book promptly appears before him projected onto one of this viewing positions. [...] Any given book of this library can thus be called up and consulted with far greater facility than if it were taken from a shelf. As he has several projection positions, he can leave one item in position while he calls up another. He can add marginal notes and comments, taking advantage of one possible type of dry photography, and it could even be arranged so that he can do this by a stylus scheme, such as is now employed in the telautograph seen in railroad waiting rooms, just as though he had a physical page before him. (Bush 1945, 121-123) 
Kern der Idee ist also auch hier ein geschickt angeordneter Index, mit dem die Mikrofilme aufgerufen und verwaltet werden können. Die auf Mikrofilm gespeicherten Dokumente haben gegenüber den herkömmlichen Archiven auf Papier den Vorteil, deutlich kompakter zu sein, da es sich um eine so weit wie möglich verkleinerte fotografische Abbildung des Originaldokuments handelt. Es ist deswegen einfacher, diese Mikrofilme platzsparend als Index zu verwalten. Allerdings ist die ganze Funktionalität auch von ebendiesem Index abhängig, denn die Auswahl von Dokumenten funktioniert mechanisch und kann nicht direkt auf die Inhalte zugreifen, wie wir es etwas bei einer Volltextsuche in digitalen Daten gewöhnt sind. Die komplexe Mechanik - „Only a small part of the interior of the memex is devoted to storage, the rest to mechanism“ (Bush 1945, 121) - verdeutlicht aber den raumgreifenden, diagrammatischen Charakter dieser Operationen, nicht unähnlich dem „großen Tisch“ und der Organisation, „sich in die Hände zu arbeiten“ der „Alphabetisatoren“ beim Thesaurus Linguae Latinae wenige Jahrzehnte davor.

Trotz der Limitationen: Der skizzierte Memex erlaubt die mechanische Umordnung der Informationen, alleine schon dadurch, dass die Mikrofilmseiten parallel über unterschiedliche Projektionen sichtbar gemacht werden. Viel deutlicher wird dies aber durch eine weitere Funktion des Memex, nämlich der Erstellung von „Trails“. Nach dem Verständnis von Bush sind das „Denkpfade“ durch die Daten, also Links, die im Prozess der Interpretation der Daten von der Benutzerin oder dem Benutzer angelegt werden. Elektromechanisch werden somit Indizes miteinander verknüpft, fotomechanisch gespeichert und somit übertragbar, beispielsweise zu weiteren Memex-Apparaten. Bush stellt sich in Folge geradezu ein neues Berufsbild vor: „There is a new profession of trail blazers, those who find delight in the task of establishing useful trails through the enormous mass of the common record“" (Bush 1945, 124).

Bushs Memex wird als Vorläufer des Hypertexts gehandelt (Morris/Tamm 1993, 173; kritisch dazu Porombka 2001, 27). Die Memex, und später der Hypertext, soll die Befreiung vom klassischen, starren Index ermöglichen und - Bush argumentiert hier durch und durch mentalistisch - ein Arbeiten ermöglichen, das eher der Funktionsweise des menschlichen Gehirns gleicht, da Dokumente durch die „trails“ immer wieder neuartig verknüpft werden können.

Bush konnte seine Idee des Memex nie umsetzen. Er entwickelte aber Konzepte für weitere Maschinen, z.B. den „Rapid Selector“, einen Vorläufer des Memex, mit dem Dokumente auf Mikrofilm nach Schlüsselwörtern durchsucht werden können, indem deren Codes ausgelesen und bei Treffern die Dokumente neu abfotografiert und ausgegeben werden. Roberto Busa, Theologe und Linguist, der für sein Vorhaben des Index Thomisticus als erster maschinelle Datenverarbeitung zur Erstellung von Konkordanzen und Wortindizes verwendete, testete 
Bushs Rapid Selector als mögliche Maschine für sein Unterfangen. Er formulierte dazu:

Of the systems I have examined up to the present time, two appeared to assure such a response: the Rapid Selector and the punched card electrical accounting machines. The former operates on microfilm, which carries, beside the photogram of the page, another, parallel one, bearing the symbols - coded in combinations of white and black spaces - of the words of that page. Before the last war Dr. Vannevar Bush of the Massachusetts Institute of Technology outlined this machine but it was completed by Mr. Ralph Shaw, librarian of the Department of Agriculture, Washington, where I was able to see it operating already in November 1949. Its principal feature is the whirlwind speed with which it explores the reels of microfilm - 10,000 photograms per minute - and instantaneously rephotographs on another microfilm strip all and only those photograms which bear a determined item. [...] I shall not give a detailed description because I thought not suitable to apply this system to the composition of concordances; I will only say that, besides not allowing automatic printing of the concordances, [...] the rapid selector necessitates on the one hand that all the cards, to be made from the sorted microfilm, be of photosensitive paper, and on the other hand all the different words and forms of each word be previously coded, for the entire text must be translated into numerical symbols by hand. (Busa 1951, 22)

Busa entschied sich für die elektronische Rechenmaschine und die Lochkarten (Busa 1951, 22). Überhaupt konnte sich Bushs Ansatz der mechanischen Datenverarbeitung nicht durchsetzen. Gleichwohl waren die grundsätzlichen Überlegungen, wie Textdokumente als Daten verwaltet und in neue Zusammenhänge gebracht werden können, zwar nicht neu, wenn man beispielsweise an die Wörterbuchprojekte denkt, aber stimulierend. Douglas Engelbart drückte 22 Jahre nach Erscheinen von Bushs Artikel in einem Brief dies wie folgt aus:

I might add that this article of yours has probably influenced me quite basically. I remember finding it and avidly reading it in a Red Cross library on the edge of the jungle on Leyte, one of the Phillipine [sic] Islands, in the Fall of 1945. (Engelbart 1992)

Engelbart widmete sich in der Folge der Konzeption und Entwicklung ähnlicher Datenverwaltungs-, Interaktions- und Kommunikationssysteme, allerdings auf Computerbasis (vgl. Abschnitt 4.2). Der analoge Weg war zu steinig für die großen Datenmengen, die Arbeiten am Thesaurus Linguae Latinae nahmen weit mehr Zeit ein als gedacht und Bushs Rapid Selector war trotz „whirlwind speed“ zu kompliziert, um sich durchzusetzen. Die analogen Methoden, Objekte zu ordnen, filtern und auszuwählen, waren der Datenflut nicht gewachsen, auch deswegen, weil diese Methoden nicht Komplexität reduzierten, sondern erzeugten. Bushs Memex sollte benutzt werden, um Trails zu finden. Um diese $\mathrm{zu}$ finden und $\mathrm{zu}$ verwalten, wird ein Trailblazer nötig: 
Dessen Aufgabe sollte sein, im vernetzten Universum von Informationen aus einer Milliarde Bücher gangbare Wege auszuspähen und Verknüpfungen zu legen. Gerade weil die Konfrontation mit der Masse von Informationen durch die Memex nicht beseitigt wird, gerade weil der Benutzer mit dieser Masse durch die ungeheure Zentralisierung erst recht konfrontiert wird, muß Bush diesen Berufsstand erfinden, der Orientierungshilfe dort geben soll, wo sie fast unmöglich geworden ist. (Porombka 2001, 45-46)

Bush scheitert, analog-automechanisch ist das nicht machbar, da dafür eine Mischung aus digitalen Daten und Hermeneutik notwendig wäre.

\subsection{Computer als Metamedium}

Dass Bush scheiterte und Busa erfolgreich war, lag also darin, dass letzterer den Computer benutzte. Bush war zumindest konzeptionell ohne Zweifel nahe dran an der Erfindung des Computers und seine Maschine und ihre Funktionsweise erinnert in vielen Teilen an die Funktionsweise eines Computers. Doch was ist der entscheidende Unterschied zwischen dem Computer und Bushs Memex?

Pierre Lévy macht in seiner Geschichte des Computers (Lévy 1998) auf den Unterschied zwischen der mechanischen Rechenmaschine und dem Computer aufmerksam. Oft wird auf Charles Babbage verwiesen, der 1837 eine Beschreibung einer Analytical Engine, einer mechanischen allgemeinen Rechenmaschine, veröffentlichte - eine Maschine, die nie gebaut worden ist, aber funktioniert hätte. Lévy wendet sich jedoch gegen die Lesart, Babbages Erfindung als Vorläufer des Computers zu sehen:

Man definiert den Computer gewöhnlich als programmierbare elektronische Rechenma-
schine mit gespeichertem Programm. Dieser letzte Begriff der Programmspeicherung ist
grundlegend, denn wie wir sehen werden, erhält der Computer erst dadurch seine flexible
Nutzbarkeit und seinen wahrhaft universalen Charakter. Nun wird die Programmspeiche-
rung aber erst dann zum technischen Vorteil, wenn eine sehr große Rechengeschwindig-
keit verfügbar ist, also erst seit dem Auftreten der elektronischen Maschinen. Der Computer
im eigentlichen Sinne war also für Babbage strikt undenkbar. Einige marginale Passagen
in den Schriften von Lady Lovelace, der wichtigsten Mitarbeiterin von Babbage, sind als
Andeutungen der Idee verstanden worden, die Programme der analytischen Maschine zu
speichern. Doch solange der interne Speicher der Maschine mechanisch, also sehr langsam
arbeitete, konnte dieser Gedanke nicht denselben Sinn haben wie für uns (Lévy 1998, 910).

In einer Tabelle vergleicht Lévy $(1998,932)$ die nötigen Zeiten für mechanische Rechenmaschinen im Vergleich zum elektronischen ENIAC, dem ersten rein elektronischen Universalrechner, um zwei 10-ziffrige Zahlen zu multiplizieren und eine Geschoßbahn zu berechnen. Babbages Rechenmaschine hätte 5 Minuten für 
die Multiplikation und 2,6 Tage für die Geschoßbahn benötigt. Die nachfolgenden mechanischen oder elektromechanischen Maschinen konnten diese Werte zwar in den Sekunden bzw. Minutenbereich drücken, doch erst die komplett elektronische ENIAC schaffte die Aufgaben in einer Millisekunde bzw. 3 Sekunden. Damit wird eine andere Dimension von Berechnung erreicht, die Lévy als entscheidend einstuft.

Doch neben der Geschwindigkeit ist ein weiterer Aspekt wichtig, mit dem der Computer sich von mechanischen Rechenmaschinen unterscheidet: Es ist - das klingt bei Lévys Zitat an, seine universelle Programmierbarkeit, die den Computer zu einem „Metamedium“ machte (vgl. dazu auch Bubenhofer 2018c). Kay und Goldberg (1977) verstehen darunter Folgendes:

\begin{abstract}
Although digital computers were originally designed to do arithmetic computation, the ability to simulate the details of any descriptive model means that the computer, viewed as a medium itself, can be all other media if the embedding and viewing methods are sufficiently well provided. (Kay/Goldberg 1977, 31)
\end{abstract}

Die Metamedialität des Computers war nicht einfach so gegeben, sondern ergab sich im Verlauf der Geschichte und hängt mit drei Typen des Schreibens mit dem Computer zusammen. Till Heilmann reflektiert Schrift und Schreiben im Zusammenhang von Computern und ordnet dieses Feld entlang einer historischen Linie:

Eine erste, wenn auch grobe, Ordnung mag dann die Betrachtung von Schreibprozessen bezogen auf den Computer als Objekt liefern: Offensichtlich kann man erstens, wie bei diesem Buch geschehen, über Computer schreiben; zweitens kann man, was für dieses Buch ebenfalls gilt, an Computern schreiben; schließlich kann man drittens für Computer schreiben - wenn man sie programmiert, bestimmte Operationen auszuführen. Die beiden letztgenannten Fälle treten in drei unterschiedlichen Konstellationen auf, welche die Geschichte des Computers als Schreibmaschine in drei einander folgende Abschnitte gliedern: das Schreiben für Computer (aber nicht an ihnen), das Schreiben für und an Computern, und das Schreiben an Computern (aber nicht für sie). (Heilmann 2012, 7-8)

Interessant an Heilmanns Ansatz seiner Mediengeschichte des Computers als ,Schreibmaschine، im Kontext der vorliegenden Arbeit ist Folgendes: Werden die Schreibprozesse mit und am Computer betrachtet, wird deutlich, wie stark der Computer von diagrammatischem Operieren geprägt ist. Dies wiederum ist eine wichtige Voraussetzung, um genauer zu verstehen, wie die algorithmische Verarbeitung von Sprachdaten und die algorithmische Generierung von Diagrammen funktioniert. Daher ist der folgende Exkurs in die Computergeschichte und die Konzeption des Computers als Metamedium wichtig (ich folge dabei Heilmann 2012). 
In den 1940er-Jahren, noch ganz unter dem Eindruck der Tradition mechanischer Rechenmaschinen, wie jene von Babbage oder Bushs Ordnungsmaschine, werden Computer als Automaten aufgefasst. Ihnen wird ein Set von Anweisungen eingeschrieben, um beliebige Eingabewerte abzuarbeiten. Sie wurden etwa zur Flugbahnberechnung von Geschossen verwendet; das dafür nötige Regelset wurde handschriftlich auf Formblättern notiert und anschließend auf Lochkarten übertragen. Die Lochkarten wiederum waren das Eingabeformat für die Rechenmaschinen und enthielten die Codes für die nötigen Operationen und zu verwendenden Speicherbereiche.

Dieser Vorgang ist beschwerlich, deshalb wurden häufig verwendete Anweisungen innerhalb dieser Regelsets in „Subroutinen“ zusammengefasst. Das war der Grundstein für die Entwicklung von Programmiersprachen. Doch bis dahin waren die Aufgaben der Formulierung der Regeln, deren Codierung für die Eingabe in den Computer und deren Abarbeitung klar voneinander abgegrenzte Aufgaben.

Dies veränderte sich in den 1960er-Jahren entscheidend damit, dass Schreibmaschinen oder Fernschreiber an die Computer angeschlossen wurden und „interactive computing“ erlaubten. Jetzt war es möglich, Anweisungen live während der Ausführung eines Programms abzusetzen und das Programm im laufenden Betrieb zu beeinflussen. Nichts anderes tun wir heute, wenn wir mit Tastatur, Maus, Bildschirmberührung etc. mit Betriebssystemen und Programmen interagieren. Doch der Anschluss der Schreibmaschine an den Computer beeinflusste nicht nur den Umgang mit Computern, sondern auch die Bedürfnisse und Möglichkeiten:

Diese neue Art des Programmierens erforderte selbst passende Programme (sogenannte Editoren) und es stellte sich schnell heraus, dass Computer nicht nur zum Schreiben von Code taugten, sondern auch dem Verfassen von technischen Berichten, Artikeln und Dokumentationen dienlich sein konnten (Heilmann 2012, 8).

Mit der PC-Revolution ab den 1970er-Jahren perfektionierte sich das Schreiben mit dem Computer und es wurden die dafür nötigen spezialisierten Anwendungen erstellt, die wir heute in Form von Textverarbeitungsprogrammen kennen. Auch Douglas Engelbarts ausgefeiltes System der Text- und Datenverarbeitung, auf das ich unten noch zu sprechen komme, gehört dazu. Damit einher gingen die Entwicklungen zu grafischen Benutzeroberflächen und zur sogenannten WYSIWYG-Darstellung - What you see is what you get -, also der Synchronisierung von Textdarstellung auf dem Bildschirm und im Druck.

Text wurde offensichtlicher Gegenstand des Computers. Aber Text war von Anbeginn in den Computer eingeschrieben: Er diente schon immer der Verar- 
beitung von Text in Form von Anweisungen, operierte schriftlich und gab Text aus, denn beim Codieren des Computers werden Werte in Form eines Systems diskreter Zeichen als Verweise auf Speicherorte „,angeschrieben“. Die schriftliche Angabe der Zahl 452, codiert auf einer Lochkarte oder in einer höheren Programmiersprache als „ $\mathrm{x}=452$ “ definiert, ist für den Computer keine Zahl, sondern ein Set von Schaltzuständen von Transistoren, also einer elektrischen Repräsentation dieser Zuweisung. Der Ausdruck „, $\mathrm{X}=452 “$ ist semantisch leer und wird rein syntaktisch übersetzt in Schaltzustände (Heilmann 2012, 65) und unterscheidet sich so nicht grundsätzlich von einer Anweisung wie „v = Haus“. Beide Ausdrücke müssen Schaltzustände auslösen, mit denen, durch weitere Textanweisungen, operiert wird.

Ich möchte dieses Operieren als diagrammatisches Operieren auffassen (was Heilmann nicht macht): Kalkulieren beim Computer bedeutet schriftliches Operieren mit Zeichen, genau so, wie wir als Menschen zum Beispiel schriftlich multiplizieren, indem wir auf Papier durch geschicktes, regelhaftes - eben: diagrammatisches - Operieren mittels Schreiben zum Ergebnis kommen (Heilmann 2012, 42 in Rückführung auf; Kittler 1989; Turing 1936). Damit wird einmal mehr deutlich, wie gerade bei der algorithmischen Verarbeitung von Text und der algorithmischen Erstellung von Diagrammen die Grenze zwischen Diagramm, diagrammatischer Operation und Daten verschwimmt.

Ein eindrückliches Beispiel des interactive computings und der Textverarbeitung gibt „the mother of all demos“ von Douglas Engelbart im Jahr 1968. ${ }^{18}$ Er und sein Team präsentierten dort während 90 Minuten live deren „oN-Line System“ (NLS) genanntes System, das alle heute gängigen Funktionen eines modernen Betriebsystems und Datenverarbeitungssystems vorwegnahm: Fenstersystem zur Ordnung von Inhalten, Hypertext, Navigationssystem, Videokonferenz, kollaborative Textverarbeitung, Grafik, Revisionskontrolle, Maussteuerung etc. ${ }^{19}$ Neu war auch, das System live zu zeigen - die „Demo“ als Format ist dabei Ausdruck der Möglichkeiten des interactive computing - ein Format, das heute bei Software völlig selbstverständlich ist. ${ }^{20}$

18 Die Aufzeichnung ist online verfügbar (SRI International 1968) und basiert auf dem Beitrag von Engelbart und English (1968).

19 Vgl. https://www.dougengelbart.org/content/view/209/448/ (letzter Zugriff: 22. 9. 2020) für weitere Informationen, insbesondere für die Verweise auf die Videoaufnahmen der Demonstration.

20 Interessanterweise haben inzwischen Softwaredemonstrationen über Screencast-Videos alle anderen Formen von Anleitungen fast komplett ersetzt. Auf Video-Portalen wie YouTube können viele Demos zu Software betrachtet werden, was eine Suche nach Software wie „Excel“, „Word Formatvorlagen“ o. ä. zeigt. 
Interessant an Engelbarts Demo sind nicht nur die Inhalte und technischen Möglichkeiten des Systems, sondern auch die Demonstration selbst. Sie war technisch äußerst aufwändig, insbesondere auch, um die Live-Demonstration filmisch festzuhalten, und geprägt durch Douglas Engelbart als Präsentator. Die Demo beginnt (nach wenigen einführenden Worten) folgendermaßen:

If in your office, you as an intellectual worker, were supplied with a computer display backed up with a computer that was alive for you all day, instantly responsible - RESPONSIVE [Lachen] - instantly responsive the reaction you had, how much value could you derive from that? Well this basically characterizes what we've been pursuing for many years in what we call the augmented human intellect research center at stanford research institute. Now the whole session is going to be devoted to trying to describe and present to you the nature of this program. But unfortunately, or fortunately, the products of this program, the technology of it be lend itself well to an interesting way to portrait it to you, so we're gonna try our best to SHOW you rather than TELL you about this program. (SRI International 1968, 2:20-3:13 meine Transkription)

Das Programm richtet sich also an, wie Engelbart sie nennt, ,intellectual worker“, also nicht an Programmierer/innen, sondern an Nutzerinnen und Nutzer für alltägliche Aufgaben, sei es im beruflichen oder privaten Umfeld. Das Programm ist ein Dienst, der „alive“ und „instantly responsive“ ist - Ausdruck des interactive computings. Engelbart verweist darauf, dass das System derart gestaltet ist, dass es sich anbietet, es zu zeigen, statt zu beschreiben, was eben daran liegt, dass es live bedient und universell genutzt werden kann, eben auch zur Demonstration seiner Funktionen.

Hier scheint mir ein feiner, aber wichtiger Unterschied zu anderen Demonstrationen von Geräten, die nicht universelle Computer sind, zu liegen: Bei der Demonstration eines Autos oder einer Nähmaschine, um zwei beliebige mechanische Beispiele zu nennen, werden die Funktionen des Fahrens (mit allem was dazugehört) und des Nähens (mit allem was dazugehört) gezeigt. Vielleicht wird auch auf die Form der Objekte verwiesen. Bei der Demonstration eines universellen Computers hingegen ist gar nicht so klar, was gezeigt wird; es wird ja nicht gezeigt, wie man schreiben, rechnen oder zeichnen kann. Es wird gezeigt, wie ich beispielsweise schreiben, rechnen oder zeichnen kann. Wenn gezeigt wird, dass geschrieben werden kann, steht dieses Zeigen im Dienst der Demonstration der universellen Operationsweisen des Computers und nicht der Funktion, Texte schreiben zu können. Es muss also die Universalität im Sinne einer Metamedialität des Computers demonstriert werden - man kann irgendetwas damit machen und dabei eben sogar eine Demo, die selbstreferentiell genau diese Funktion hat, nämlich sich selbst zu demonstrieren. 
Engelbart fährt fort und zeigt zunächst die Möglichkeiten der Textverarbeitung, wovon unten nur ein kleiner Teil wiedergegeben ist:

\begin{abstract}
So in my office I have a console like this and there are twelve others that our computer supplies and we try nowadays to do our daily work on here. So this characterizes a way I could sit here and look at a completely blank piece of paper - that's where I start many projects. So with my system that's a good start. I sit here and say I'd like to load data in. So I'm putting in an entity called a statement and that's full of other entities called words. And if I make a mistake I can back up a little bit. So I have a statement with some entities words and do some operations on these. I can copy a word. I can say that word like copy after itself. In fact that pair of words I like to copy after it selves. I can just do this a few times and get a bit of material there. And there are other entities like text. I can say after there I'd like to copy from that entity point to that point and copy it. (SRI International 1968, 3:28-4:41, meine Transkription)
\end{abstract}

Während des Sprechens bedient Engelbart die drei Eingabeinstrumente Tastatur, Maus und Befehlstasten und zeigt live, wovon er erzählt. Der Inhalt dieses Teils ist das Schreiben, Löschen und Kopieren von Wörtern und Absätzen - also aus heutiger Sicht keine weltbewegenden Funktionen (im Gegensatz zu den später noch gezeigten Operationen, die selbst aus heutiger Perspektive noch überraschen). Das Operieren mit Text auf einer daten- und programmiertechnischen Grundlage wird bei den Ausführungen aber sehr deutlich: es ist von „entities“, „statements“ und „operations“ die Rede. Im Vergleich zu einer Schreibmaschine sind die Operationsmöglichkeiten hier schon wesentlich vielfältiger, da Textsegemente verändert werden können. Im weiteren Verlauf der Demo zeigt Engelbart, wie eine Einkaufsliste erstellt werden kann, die umsortiert, hierarchisiert und gar in einen grafischen Vektor konvertiert werden kann, der den zu gehenden Weg zu den Geschäften in optimaler Reihenfolge beschreibt (vgl. Abbildung 32, s. u.).

Im weiteren Verlauf der Demo fallen zahlreiche Begriffe, die Operationen zur Text- und Datenmanipulation beschreiben und klassische diagrammatische Operationen sind, wie ich sie in den Abschnitten 3.1 und 4.1 bereits vorgestellt habe und in Kapitel 5 noch ausführlicher beschreiben werde: „do some operations on it“, „,constructing views“, „organize, categorize, subcategorize [the list]“, „so we have this feature to structure our material“, „make different views [...] modifying the structure" (SRI International 1968).

Deutlich wird dabei auch, dass die Daten immer wieder in neuen Ansichten präsentiert werden und so neue Einsichten ermöglichen. Eine bestimmte Ansicht „makes it very nice for studying“ oder die grafische Darstellung des optimalen Einkaufsweges aufgrund der Einkaufsliste ermöglicht es, ,to see the route I have to go [...] that's my plan for getting home tonight“.. Und man interagiert mit dem System - ,just point on it to see“ - und ermöglicht so eine neue Sicht. 

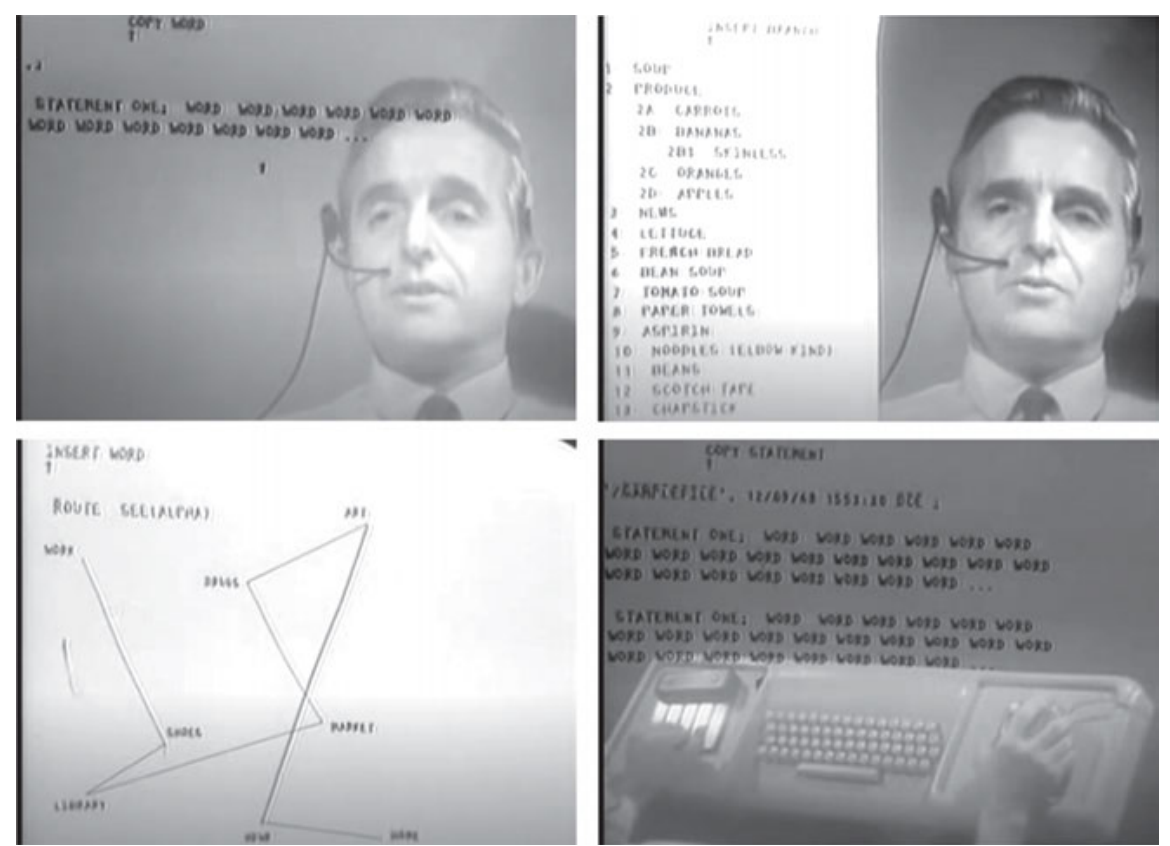

Abb. 32: Standbilder aus der NLS-Demo von Douglas Engelbart von 1968 (SRI International 1968)

Dieses Beispiel zeigt nun die bereits weiter oben von Kay und Goldberg definierte Metamedialität des Computers. Sie zeigt sich gerade auch im Format der Demo, die sich durch das Zeigen der Metamedialität viel besser dafür eignet als die Beschreibung. Wenn man so will ist eben auch die Demo ein durch das Metamedium Computer erzeugtes Medium.

Die Metamedialität ergibt sich aufgrund der drei Grundfunktionen des Computers, nämlich der Codierung, Algorithmisierung und Formatierung (Heilmann 2012, 195), mit denen Daten gespeichert, übertragen und verarbeitet werden. Mit der Codierung werden Eingaben in Transistorzustände (und damit ins Digitale) übersetzt. Die Algorithmen operieren mit den Eingaben und Daten und durch die Formatierung werden die abstrakten Transistorzustände wieder sicht- und deutbar gemacht. Keine dieser Repräsentationen ist die „eigentlich richtige“ Repräsentation der Transistorzustände; es gibt unendlich viele. Programmcode, also Text, sorgt für die Formatierung der Daten und so - handelt es sich bei den Daten um Text - gelangen wir zur Erkenntnis, dass damit 
die Formatierung von Texten eine Sache der Beschreibung von Text durch Text [ist]. Zum ersten Mal in der Geschichte des Schreibens bestimmen damit Texte über die ,Materialität* der Schrift und nicht umgekehrt die Materialität der Schrift über die Gestalt von Texten (Heilmann 2012, 239).

Traditionell bestimmte also die Materialität von Schrift (auf Papier gedruckt, in Holz gekerbt, mit Pinsel und Tusche gemalt etc.) über die Gestalt von Texten. Beim Computer ist es anders: Text (als Programmcode) weist an, den Text (textuelle Daten), repräsentiert durch elektronische Schaltzustände, in Schrift (dargestellten Text) zu materialisieren - auf Bildschirmen in verschiedenen Formen, zu übertragen, auszudrucken. Was ändert sich damit?

Es ergibt sich ein riesiges Potenzial an Transformationen von Text in unterschiedliche Formen. Mit Jäger gesprochen (vgl. Abschnitt 3.1) sind also vielfältige Transkriptionen möglich, die jeweils auch eine neue Medialität mit sich bringen. Dazu kommt, eben durch die Form des interactive computing, ein rekursiver Prozess der Textbeeinflussung: Solange der Text nicht ausgedruckt wird, können diese Transformationsprozesse, veranlasst durch Text, ständig und live wiederholt werden. Durch Text (als Programmcode) kann veranlasst werden, dass ein Text in Textseiten aufgeteilt auf dem Bildschirm ausgegeben und damit Blättern in einem Dokument simuliert wird; oder aber der Text wird als Frequenztabelle ausgegeben oder in eine völlig andere Form gebracht. Dahinter stecken diagrammatische Operationen, die manchmal auch grafisch als solche sichtbar werden, etwa wenn die Frequenztabelle mit Klick sortiert werden kann. Aber das ist sozusagen nur die Spitze des Eisbergs, denn alle Transformationsprozesse, die ausgelöst werden, und sei es auch „nur“ das Löschen eines Wortes, sind diagrammatische Prozesse, also diagrammatische Transformationen, die auch bei Operationen mit grafischen Diagrammen vorkommen können. Dies bedeutet eben auch, dass damit diagrammatisches Operieren mehr ist als die Interaktion, z. B. über eine grafische Benutzeroberfläche, mit dem grafischen Diagramm als Ergebnis einer bestimmten Datenformatierung. Interaktion umfasst alle diagrammatischen Operationen, zu denen der Computer fähig ist, also alle algorithmischen Transformationen und Formatierungen von Daten. Das sind Vorgänge, derer sich eine Nutzerin / ein Nutzer, z. B. bei der Arbeit in einer Software zur Erzeugung von Diagrammen, kaum bewusst ist.

Anders ist das etwa bei einer Skizze auf Papier: Zwar kann auf Papier diagrammatisch operiert werden, etwa indem ein geometrischer Beweis geführt oder eine Karte gelesen wird, die materiellen Vorbedingungen aber, Papier, Stift, können nicht durch den gleichen Operationstyp, also durch Zeichenmanipulation, verändert werden. Beim Computer hingegen ist die Wahl der Form der Materialisierung Teil des Möglichkeitsraums diagrammatischer Operationen, etwa indem ich zur Materialisierung einen Bildschirm, einen Papier- oder gar einen 
3D-Drucker verwende. Insofern kann man alle Operationen mit digitalen Daten im Computer als diagrammatische Operationen auffassen. Der Computer wird dann zur diagrammatischen Maschine.

Diagramme sind also nicht bloß „Bilder“, die durch Transformationen von Daten entstanden sind, sondern alle damit verbundenen Transformationsprozesse in den digitalen Daten selbst haben ebenfalls diagrammatischen Charakter.

In Abschnitt 4.1 habe ich beschrieben, wie die Zettel eines physischen Zettelkastens für ein Wörterbuch von Hand sortiert werden, indem sie systematisch auf einem großen Tisch ausgebreitet werden. Diagrammatische Operationen dieses Typus kommen im Computer dauernd vor und können in ständiger Interaktion ausgelöst oder völlig neu gestaltet werden, etwa indem ich ein eigenes Script schreibe, das die Transformationen auf eine neue Art durchführt. Eine interaktive Visualisierung ist erst dann auch in einem diagrammatischen Sinn vollständig interaktiv, wenn auch alle Interaktionen mit den Daten möglich sind. Also wenn die digitale Verarbeitung und algorithmischen Manipulationen ebenso dazugehören wie die Beeinflussung der visuellen Darstellung. Das ist der Kern des „interactive computing“.

\subsection{Generische Anweisungen}

Der Computer als Metamedium ist natürlich auch in der Lage, Diagramme zu zeichnen, das bedeutet, grafisch sichtbar zu machen. In Engelbarts Demo (vgl. Abschnitt 4.2) sind dies Diagramme in Form von (hierarchischen) Listen, aber auch ein prototypischeres Diagramm, der grafische Vektor, nachdem der Weg zu den Geschäften in einer bestimmten Reihenfolge zu gehen ist, um die Einkaufsliste optimal abarbeiten zu können. Die Unterscheidung zwischen Diagramm und Nichtdiagramm ist dabei unscharf, was eben am Metamedium Computer liegt, mit dem eine elektronisch codierte Information (Transistorzustände) in beliebige Ansichten transkribiert werden kann. Denn ob ein Text, den wir im ausgedruckten Zustand als deutlich weniger diagrammatisch oder gar nicht diagrammatisch interpretieren würden, auf dem Computerbildschirm als Text oder Liste materialisiert wird, hängt eben von der Formatierung ab. Die elektronisch codierte Information kann derart sein - oder kann so algorithmisch bearbeitet werden -, dass daraus eine Gliederung formatiert werden kann, etwa in Form von Absätzen. Der gleiche Text kann aber auch als Endloszeile ohne Absätze, links- oder rechtsbündig, zentriert oder im Blocksatz, als Liste, evtl. sogar hierarchisch gegliedert, formatiert werden, je nachdem, welche Informationen elektronisch dazu codiert sind. Die diagrammatische Erscheinungsform gibt also nicht den vollen Umfang seiner Codierung wieder. 
Diese Codierung kann sehr unauffällig sein; insbesondere bei Text ist dies der Fall, wenn man sich etwa vor Augen führt, dass jedes codierte Zeichen eindeutig und diskret als ebendieses Zeichen codiert werden muss, dass ein Leerzeichen zwischen Wörtern ebenso ein eindeutig codiertes Zeichen ist (in ASCII z. B. Code 32, in Unicode U+0020) und nicht einfach Leere, dass ein Absatz ebenfalls mit einem diskreten Zeichen codiert ist und deshalb auch genau definiert ist, wie viele Leerzeilen zwischen zwei Absätzen vorhanden sind. Bei handschriftlich verfasstem Text ist diese Eindeutigkeit auf keiner Ebene in der Form vorhanden. Wie groß ein Abstand zwischen zwei Wörtern ist, überhaupt, wo die Wortgrenzen liegen und welche Zeichen überhaupt vorhanden sind, muss gedeutet werden und es gibt keine Möglichkeit zu eruieren, was die richtige Deutung ist - man kann nicht hinter den Text sehen, wie das beim elektronisch gespeicherten Text der Fall ist.

Beim traditionellen Buchdruck hingegen, durch das System der beweglichen Lettern, liegt eine ähnliche Situation wie beim elektronisch gespeicherten Text vor, wobei die Codierung dort eher eine grafische, denn eine logische ist: Für die verschiedenen Spatien verschiedener Breite existiert zwar normalerweise entsprechendes Blindmaterial, also nicht-druckende Lettern. Um etwa Blocksatz zu erzeugen, wird zwischen die Wörter (oder Buchstaben) jedoch nach Bedarf beliebig viel Blindmaterial eingefügt, so dass die Information, dass eigentlich nur ein Leerzeichen zwischen den Wörtern gemeint ist, verloren geht. Anders beim Computer, wo auch bei Blocksatz und einem Anpassen der Spatien zwischen den Wörtern die Information „ein Leerzeichen“ erhalten bleibt, für die Formatierung aber die Darstellung des Leerzeichens modifiziert wird.

Beim Computer werden Buchstaben als Zeichen, Wörter, Absätze, Texte etc. als Entitäten codiert, so dass eben mit diesen Entitäten operiert werden kann. Dies ist natürlich der Gewinn der algorithmischen Bearbeitung und Analyse von digitalen Daten mit dem Metamedium Computer und löste alleine auf Textebene eine Reihe von neuen Möglichkeiten der Textproduktion und -rezeption aus (vgl. dazu ausführlich Lobin 2014, 98-153).

Aus analytischer Perspektive zielt man nun auf die hinsichtlich einer Methode systematische Transformation der Daten, um neue Perspektiven darauf zu gewinnen. Bei Busa (vgl. Abschnitt 4.1) war dies eine technische Herausforderung und die gewünschten diagrammatischen Operationen mussten auf einer sehr programmiertechnisch nahe Weise abgebildet werden. Je abstrakter die Programmiersprache ist, mit der diese Operationen ausgedrückt werden, desto unsichtbarer sind die grundlegenden elektronischen Operationen, die dafür nötig sind. Bei einer Sortierfunktion muss man sich keinen Sortieralgorithmus überlegen, geschweige denn Speicherbereiche reservieren, füllen und darauf zugreifen, wie das beim Schreiben von sogenanntem „Maschinencode“, also Hardware- 
nahem Programmieren der Fall ist. ${ }^{21}$ Stattdessen muss man höchstens die entsprechende Funktion aufrufen. Die Medialität der Hardware des Computers wird somit - mit Jäger gesprochen (vgl. Abschnitt 3.1) - transparent. Dafür wird das enorme Potenzial an möglichen Datentransformationen sichtbar, das dann eben genutzt werden kann, um neue Ansichten, also diagrammatische Ansichten, zu erzeugen.

Fragt man nun nach dem Unterschied zwischen Diagrammen, die mit und solchen, die ohne Computer erstellt worden sind, scheint zunächst das Vorhandensein eines Algorithmus entscheidend zu sein. Aber was ist ein Algorithmus und was sind nicht-algorithmische Diagramme?

Ein Algorithmus ist ein bestimmtes Regelset zur Lösung eines Problems. Dieses Regelset kann schematisch immer wieder auf neue Ausgangsdaten angewandt werden. Ein Algorithmus ist nicht an einen Computer gebunden, sondern kann auch auf nicht-digitale Gegenstände und in nicht-digitaler Form angewandt werden.

Da ein Diagramm ein Schema ist, das immer wieder auf neue Daten hin angewendet werden kann, sind nicht-algorithmische Diagramme schwer denkbar. Worauf die Frage nach dem Unterschied mit und ohne Computern erstellten Diagrammen also zielt, ist der Unterschied zwischen einem Diagramm, das über Programmcode und einem, das manuell erstellt worden ist. Ob letzteres mit dem Computer erstellt worden ist, ist unerheblich: Es kann ein Grafikprogramm o. ä. eingesetzt werden, um ein Diagramm auf dem Computer zu zeichnen - allerdings

21 Eine Liste zu sortieren ist für einen Computer keine triviale Angelegenheit. So muss definiert werden, nach welchen Kriterien sortiert werden soll: numerisch, alphanumerisch, alphabetisch; sodann gibt es unterschiedliche Strategien, zu sortieren: Der Algorithmus kann z. B. darin bestehen, die Liste der zu sortierenden Elemente durchzulaufen und das kleinste Element auszuwählen und an den Anfang zu stellen. Danach wird die Liste erneut durchlaufen und wiederum das kleinste Element ausgewählt und an die zweite Position gestellt, etc. Dieses Verfahren nennt sich „Selectionsort“. Oder aber mit der Methode „Quicksort“ wird ein Element ausgewählt, das als Vergleichselement dient. Nun wird für jedes Element entschieden, ob es kleiner oder größer als das Vergleichselement ist. Kleinere kommen auf die linke, größere auf die rechte Seite. Nun werden links und rechts des ersten Vergleichselements neue Vergleichselemente ausgewählt und wieder wie zuvor verfahren. Dies wird wiederholt, bis es keine neuen Vergleichselemente mehr gibt. Es gibt zahlreiche weitere Verfahren, die jeweils Vor- und Nachteile bezüglich Geschwindigkeit und Eignung für große Datenmengen haben (Knuth 1998). Wird ein solcher Algorithmus in einer maschinennahen Programmiersprache implementiert, muss die Verwendung des verfügbaren Speichers genau geplant und entschieden werden, wann welche Informationen wo gespeichert werden sollen. Bei modernen Programmiersprachen oder gar der Anwendung einer Sortierfunktion in einem Programm wie Excel muss man sich nicht mal mehr Gedanken über den Sortieralgorithmus machen. 
so, dass nicht ein generisches Regelset definiert worden ist, dies zu erzeugen, sondern indem Linien, Flächen und dergleichen gezeichnet werden.

Der Unterschied liegt in der Semantisierung der erzeugten grafischen Elemente: Zeichnet man mit dem Computer ein Balkendiagramm so, dass man z. B. in einem Grafikprogramm Flächen mit bestimmten Maßen definiert und darstellen lässt, dann haben diese Flächen für den Betrachter oder die Betrachterin wahrscheinlich die Bedeutung, Balken in einem Balkendiagramm zu sein. In der elektronischen Codierung jedoch handelt es sich bei den Flächen bloß um das: Flächen. Also die Definition der x- und y-Werte von vier Punkten, die ein Rechteck ergeben.

Wenn ein Balkendiagramm mit Programmcode erstellt wird, sind die Flächen als Balken des Diagramms codiert. Der Code beinhaltet die Information, dass diese Zahlenwerte repräsentieren, dass alle Balken des Diagramms in Relation zueinander stehen etc. Die Balken sind also als Entitäten einer größeren Entität, einem Diagramm, definiert.

Bei mit Programmcode erstellten Diagrammen sind die Diagramme Ergebnis einer ununterbrochenen Kette von Transformationen oder diagrammatischen Operationen derselben Art, nämlich der Art, wie sie ein Computer vollziehen kann und derart, dass jede Transformation rückgängig gemacht werden könnte, um die ganze Genese der Transformationskette zu rekonstruieren. Diese Kette wird unterbrochen, wenn man, basierend auf Zahlenwerten, in einer (auch digitalen) Tabelle ein Diagramm manuell zeichnet (egal ob mit Computer oder auf Papier) - dann kommt es (mit Jäger) zu einer intermedialen Transkription und (mit Bolter/Grusin) einer Remediatisierung (vgl. Abschnitt 3.1).

Wenn also, wie es in den Visual Analytics z. B. der Fall ist, mit Programmcode Diagramme erstellt werden, handelt es sich um eine Folge von intramedialen (und nicht intermedialen) Transkriptionen mit schematischem Charakter. Dies bedeutet auch, dass diese Transkriptionen der medialen Logik folgen - von der ersten Transkription auf elektronischer Ebene bis zur Darstellung auf einem Bildschirm.

Diese mediale Logik gilt es im Folgenden nun noch aus einer bestimmten Perspektive zu beleuchten, nämlich mit einem kulturellen Zugriff auf die Praxis des Programmierens.

\subsection{Coding Cultures}

In der bereits weiter oben erwähnten Studie zur Erfindung des Computers von Pierre Lévy (1998) wirft der Autor die Frage auf, ob die Geschichte des Computers als Technikgeschichte von der mechanischen Maschine zu elektromechanischen 
und schließlich zur elektronischen, erzählt werden muss. Er verneint diese vereinfachende Sicht:

\begin{abstract}
War die Entwicklung des Mikroprozessors die eigentliche „Ursache“ für den Erfolg des Personal Computers? Nein, sie war nur ein Ereignis unter anderen, das seinen Sinn und seine Dynamik erst im Rahmen eines Kampfes gegen die Riesen der Informatikbranche gewann. Nennen wir aus dem Sammelsurium der Faktoren, die sich die Gründer der ersten Mikrocomputer-Firmen zunutze machten: Die Programmiersprache Basic; Schnittstellen, die auch von Leuten benutzt werden konnten, die keine professionellen Informatiker waren; die Gegenkulturbewegung, die ihren Höhepunkt in den siebziger Jahren in den Vereinigten Staaten erreichte; Risikokapital-Gesellschaften auf der Jagd nach raschen Profiten; und so weiter. [...] 1976 verband IBM mit dem Mikroprozessor etwas anderes als Apple; es waren in der Tat inkompatible „Welten“, das heißt unterschiedliche Netze von Allianzen, in die er hier und dort eingebunden war. Dieses Beispiel legt den Gedanken nahe, daß „kausale“ Erklärungen [...] in der Geschichte der Technik manchmal ohne Belang sind. Interessanter scheint es, hervorzuheben, in welcher Weise die Akteure Situationen, technische Anordnungen und soziale Kräfte zum eigenen Vorteil deuten, „umwidmen“ oder „kapern“ (Lévy 1998, 919-920).
\end{abstract}

Praktiken des Umgangs mit Technik sind also genau so relevant - oder gar relevanter als technische Entwicklungen, um die Bedeutung computertechnischer Verarbeitung $\mathrm{zu}$ verstehen. Dies gilt es zu berücksichtigen, wenn die mediale Logik des Computers im Hinblick auf diagrammatische Transformationen ausgeleuchtet werden soll. Dies möchte ich im Folgenden tun, indem ich die Praxis des Programmierens für die Erstellung von Diagrammen in den Blick nehme. Es stellt sich dabei beispielsweise die Frage, ob die Wahl einer bestimmten Programmiersprache oder Programmierumgebung wie R, Javascript oder Excel einen Einfluss auf die diagrammatische Praxis hat. Und weiter, ob solche vermeintlich rein technischen Entscheidungen bei der Anwendung solcher Diagramme, also der Erstellung und Deutung, von Belang ist.

Ich plädiere dafür, diese kulturellen Entstehungsbedingungen unbedingt mitzudenken, wenn man mit Visualisierungen arbeitet, die mit Computern erstellt worden sind. Es ist meines Erachtens notwendig, die „Coding Culture“, in deren Kontext der Programmiercode entsteht, mitzubedenken. Als Coding Culture verstehe ich eine Kultur von Praktiken des Programmierens. Solche Coding Cultures sind dehalb wichtig, weil die Entscheidung für die eine oder andere Programmiersprache, für den einen oder anderen Algorithmus, für die Verwendung dieser oder der anderen Funktion primär keine technische Entscheidung ist, sondern eine Entscheidung die in Abhängigkeit der Coding Culture steht, in der man sich bewegt. 


\subsubsection{Praxis des Programmierens}

Die Software Studies machten bereits vor einigen Jahren auf die kulturelle Verfasstheit von Software aufmerksam (Fuller 2003; Mackenzie 2006). Software spiegelt bestimmte kulturelle Praktiken wider und reproduziert diese gleichzeitig. Grafische Benutzeroberflächen, die „eine ganze Maschine ihren Benutzern entziehen“ (Kittler 1993, 233), ermöglichen und verunmöglichen gewisse Praktiken, noch stärker als es Programmiersprachen tun, die bestimmte Operationen zulassen oder eben nicht. Die Art und Weise, wie Daten in Datenbanken repräsentiert werden, steht in Abhängigkeit kultureller Übereinkünfte zu Wissensrepräsentation (Dourish 2014; Manovich 2002). So besteht beispielsweise im Zuge von Open-Data-Bewegungen inzwischen (zumindest in Demokratien) mehr oder weniger Konsens darüber, dass öffentliche Daten auch öffentlich verfügbar sein sollen - und zwar in strukturierter, maschinenlesbarer Form. Die Möglichkeit, die Daten in gedruckter Form einsehen zu können, würde dem Ideal der Open-DataIdee nicht genügen.

Einfach - also meist: frei - verfügbare und handhabbare Software beeinflusst die Praxis der wissenschaftlichen Visualisierungen erheblich, wobei disziplinäre Unterschiede offensichtlich sind. Während beispielsweise in vielen naturwissenschaftlichen Fächern und auch in der Computerlinguistik oder in den Digital Humanities die Open-Source-Programmierumgebung „R“ für statistische Analysen und Visualisierungen weit verbreitet ist, wird in den Sozialwissenschaften noch stärker das kommerzielle Softwarepaket „SPSS“ verwendet. Und in den Geisteswissenschaften, so auch in der Linguistik, kommt eher Excel zum Einsatz. Diese Selektionspraxis ist natürlich stetigem Wandel unterworfen und bestimmte Gruppen und Denkkollektive innerhalb dieser Disziplinen verwenden (in der Tendenz) andere Werkzeuge.

Ich möchte den Blick aber über Software hinaus lenken und auch Programmiersprachen in den Blick nehmen, da anzunehmen ist, dass auch die Implementierung von Software in bestimmten Programmiersprachen abhängig von Coding Cultures ist und deshalb eine Auswirkung auf die Ergebnisse, also die Software, hat.

Für die kommerzielle Welt sieht der Informatikjournalist Paul Ford (2015) die Wahl der Programmiersprache als ,the most important signaling behavior that a technology company can engage in“ (Ford 2015) und nennt die überspitzten Klischees, mit denen unter Programmierer/innen die Sprachen bedacht werden:

Tell me that you program in Java, and I believe you to be either serious or boring. In Ruby, and you are interested in building things quickly. In Clojure, and I think you are smart but wonder if you ship. In Python, and I trust you implicitly. In PHP, and we sigh together. In 
$\mathrm{C}++$ or $\mathrm{C}$, and I nod humbly. In C\#, and I smile and assume we have nothing in common. In Fortran, and I ask to see your security clearance. These languages contain entire civilizations. (Ford 2015)

Es lassen sich viele solcher Zuschreibungen finden (sie sich natürlich oft widersprechen). Der Linguist und Erfinder der Programmiersprache Perl, Larry Wall, hielt beispielsweise an der Konferenz „LinuxWorld“ eine bekannte Rede mit dem Titel „Perl, the first postmodern computer language“ (Wall 1999), wo er auf zwar unterhaltsame, jedoch plausible Weise argumentiert, dass sich Perl gegen vier Kulte richte, die die Informatik beherrschten: „spareness“, „originality“, „seriousness“ und „objectivity“. Perl sei insofern konträr, als dass es Lösungsdogmen vermeide und Diversität zulasse:

Perl programming is unabashedly genre programming. It has conventions. It has culture. Perl was the first computer language whose culture was designed for diversity right along with the language. (Wall 1999)

Diese Kulte, die man als Topoi auffassen kann, werden auch von Fuller (2003, 15) beschrieben, der die ,idealist tendencies in computing“ beschreibt, mit der der Purismus von Zahlen mit Schönheit gleichgesetzt werde. Dieser Topos würde zum Dogma des „ästhetischen Programmierens“ führen, das über soziale Kontrolle durchgesetzt wird. „Märchenhafte“ („fabulatory“) Programmieransätze wären aber interessanter:

Numbers do not provide big answers, but rather opportunities to explore further manifold and synthetic possibilities-that is to say, they provide access to more figures. (Fuller 2003, 16)

Neben dem Purismus-Topos wird auch vom Utilitarismus-Topos gesprochen, der die Informatik beherrscht (Goffey 2014, 21), aber auch Technologie generell prägt (Böhme 2006, 55; vgl. auch Bubenhofer 2018c). Diese Topoi fließen dann in die Programmierung von Software mit ein.

Im Abschnitt oben (4.3) habe ich argumentiert, dass programmiertechnisch erstellte Visualisierungen intramediale Transkriptionen seien, da sie alle auf denselben elektronischen Grundlagen stattfinden. Diese Sicht berücksichtigt jedoch noch nicht, wie die Transformationen in formatierter Form erscheinen - auch in einem komplexen Zusammenspiel unterschiedlicher Transformationen als Software - und gelesen werden. Aus Sicht der Software Studies merkt auch Andrew Goffey an, dass Software mit ihrer eigenen Medialität virtuelle Welten modellieren würde: 
Strictly speaking, many of the things that software models are not real-world processes at all, they are things that are brought into being by computational technologies themselves. (Goffey 2014, 34)

Goffey bezeichnet dies als „demiurgische Qualität“, als schöpferische Kraft von Software, die etwas erzeugt, was vorher nicht existierte (Goffey 2014, 35). Der Code aber, der zu Software führt, wird unsichtbar, sobald Software benutzt wird. Ihr ist die Praxis der Codeerzeugung nicht mehr anzusehen, insbesondere nicht die bunte Mischung verschiedener Codetypen, Tricks und Basteleien - Goffey spricht von „Bricolage“:

\begin{abstract}
clever tricks with assembly language, manipulating the side effects of an algorithm, devising workarounds, the smoke and mirrors of user interface design, and so on. The virtuoso smarts of programming practice that get software working can of course eventually be explained logically if the code compiles, the app works and users accept it. But in doing so, the process disappears into the product and the experimenting, the ,bricolage' gets forgotten. (Goffey 2014, 35)
\end{abstract}

Die Tricks und Bastellösungen während des Programmierens verschwinden also im Endprodukt, bei dem nur noch entscheidend ist, ob es funktioniert oder nicht. Dies ist natürlich der Fall, da Software mit der Benutzung eine eigene semiotische Qualität erhält, mit der nun eben ein Mensch und nicht mehr die Maschine, interagiert und ihr seine eigenen Sinnzuschreibungen vornimmt. Damit kehrt die „messiness of the world“ (Goffey 2014, 37) zurück - oder, mit Jäger gesprochen, es entsteht eine neue Transkription. Trotzdem sind in der Software die Programmierpraktiken, die zu ihrer Erstellung führten, eingeschrieben, aber nicht sichtbar. Es sei denn, ein Programmierfehler tritt auf: Dann wird die Medialität der Software für einen kurzen Augenblick als Störung wahrnehmbar.

Obwohl die Programmierpraktiken nicht mehr sichtbar sind, beeinflussen sie jedoch die Form und den Möglichkeitsraum der Operationen sowie den Bedeutungsraum (,software is a semiotic artefact“ Goffey 2014, 36) der Software. Wenn man sich mit Computern erstellte wissenschaftliche Visualisierungen generell der letzten Jahrzehnte ansieht, dann sind Trends und Moden wie auch disziplinäre Eigenheiten sichtbar, obwohl es zu jedem Zeitpunkt immer eine ganze Palette von alternativen Erstellungsweisen gegeben hätte. Diese Trends und Moden sind eben $\mathrm{u}$. a. ein Ergebnis von unterschiedlichen Programmierpraktiken, die ich „Coding Cultures“ nenne.

Eine sehr umfassende Coding Culture ist das Hacking, das wiederum eine Vielzahl weiter unterscheidbarer Coding Cultures umfasst. „Hacking“, verstanden als Tätigkeit, die nicht primär ein konstruktives Ziel erfüllt, sondern dem Selbstzweck dient (Levy 2010, 10), ist ein wichtiger Einflussfaktor der Compu- 
tergeschichte. Ich möchte dieses Thema an dieser Stelle nur kurz streifen (vgl. ausführlich Coleman 2012; Levy 2010) und darauf hinweisen, dass Prinzipien wie transparenter Code, Open Source, Weitergabe von Code etc., die bei Software und der Programmierung in der Wissenschaft eine wichtige Rolle spielen, auf die Hackerkultur zurückgehen. Benutzt man also Open Source-Software, sind ein Teil dieser Kultur und bestimmte Coding Cultures der Software eingeschrieben und fließen auch in die Benutzungspraxis mit ein. Dies ist noch stärker der Fall, wenn basierend auf solchen Systemen neue Software erstellt wird, wie das beispielsweise für die Erstellung von Visualisierungen mit R, Javascript, Perl, Python o. ä. der Fall ist.

Ein Beispiel für die Wirkung der Coding Cultures soll an dieser Stelle R geben: Im Web sind zahlreiche Statements zu finden, die sich zur „Philosophie“ dieser Programmiersprache äußern, beispielsweise in einem Blogeintrag:

The visualizations you can create in $\mathrm{R}$ are much more sophisticated and much more nuanced. And, philosophically, you can tell that the visualization tools in R were created by people more interested in good thinking about data than about beautiful presentation. (The result, ironically, is a much more beautiful presentation, IMHO.) $)^{22}$

Die Begründung für die Benutzung von R geschieht also nicht auf einer technischen, sondern einer ideologischen Ebene - apostrophiert als „philosophically“. Hier ist der Purismus-Topos am Werk, der eine Gleichsetzung von ästhetischem Purismus und Denken postuliert. „Gutes Denken“ über Daten würde durch die Benutzung von R gefördert und führe zu „schönen“ Visualisierungen, ohne dass dies intendiert wäre. Ähnliche Gleichsetzungen finden sich beispielsweise beim insbesondere in der Wissenschaft verbreiteten Satzsystem TeX bzw. dem darauf basierenden LaTeX, das Textinhalt und Layout konsequent trennt und dafür verspricht, beim Setzen für ein perfektes Layout zu sorgen. ${ }^{23}$

Wenn man nun, als an Programmierphilosophien uninteressierte Forscherin oder Forscher, mit $\mathrm{R}$ zu arbeiten beginnt, wird man auf der Suche nach Lösungen für die unweigerlich auftretenden Probleme im Netz verschiedene Lösungsmöglichkeiten finden. Da diese Lösungsmöglichkeiten oft in Web-Diskussionsforen präsentiert werden, existieren dazu meist eine Reihe von Kommentaren, bis hin zu ausufernden Diskussionen über das Für und Wider der verschiedenen Lösungsmöglichkeiten. Wohlgemerkt: Alle diese Lösungen führen jeweils zum selben

22 http://www.michaelmilton.net/2010/01/26/when-to-use-excel-when-to-use-r/ (24. 8. 2015)

$23 \mathrm{TeX}$ wurde vom Informatiker Donald E. Knuth aus Unzufriedenheit über die Satzmöglichkeiten für sein mehrbändiges Werk „The Art of Computer Programming“ (der erste Band erschien 1968 und die Reihe ist noch nicht abgeschlossen; Knuth 2011) selbst entwickelt. 
I want to conditionally subset a dataframe without referencing the dataframe. For example if I have the following:

long_data_frame_name <- data.frame $(x=1: 10, y=1: 10)$

I want to say:

subset $<-$ long_data_frame_name $[x<5$,

But instead, I have to say:

subset $<$ - long_data_frame_name[1ong_data_frame_name $\$ x<5$, ]

plyr and ggplot handle this so beautifully. Is there any package that makes subsetting a data frame similarly beautiful?

dataframe subset

share improve this question

asked Nov 1 '12 at 15:00

Figh Ben Haley

$1,909 \cdot 1 \cdot 10 \cdot 16$

3 This is what subset does. - Matthew Plourde Nov 1 '12 at 15:05

3 Beauty is in the eye of the beholder. :-) You may find with more lovely. - Carl Witthoft Nov 1' '12 at 15:07

@Carl I don't see how with would apply in this case. That being said, though, I think it's hard to write beautiful R code without using with and within . - Matthew Plourde Nov 1'12 at 15:15

with was made for this. - Roman Luštrik Nov 1 '12 at 15:19

1 @Roman How so? You'd still have to type the name of the data. frame twice. - Matthew Plourde Nov 1 '12 at 15:23

I agree with @mplourde: with with, you would still need subset <-

with (long_data_frame, long_data_frame_name $[x<5$,$] ), which doesn't save much (it only becomes$

Abb. 33: Beispiel einer Diskussion auf der Plattform „Stackoverflow“ unter dem Titel „Is there a better syntax for subsetting a data frame in R?“, wobei die Kategorie „Schönheit“ eine zentrale Rolle spielt (https://stackoverflow.com/questions/13179792/is-there-a-better-syntax-for-subsetting-a-data-frame-in-r, 22.9.2020)

Resultat - lösen also das Problem. Aber sie tun es auf unterschiedliche Weisen, wobei offensichtliche Kriterien die Länge des Codes oder die Abhängigkeit oder Unabhängigkeit von bestimmten Programmbibliotheken sind. Dabei fallen Argumente zur Eleganz, Ästhetik oder Sauberkeit der Lösungen und es wird deutlich, wie ideologisch geprägt die Diskussion ausfällt. In Abbildung 33 ist eine solche Diskussion abgebildet, wo ein Programmierer nach einer „schönen“ Lösung für ein Problem sucht, das er aber auf eine „unschöne“ Weise schon gelöst hat.

Wenn also selbst Lösungswege innerhalb derselben Programmiersprache ideologisch diskutiert werden, ist das beim Vergleich verschiedener Programmiersprachen oder Softwareplattformen noch ausgeprägter der Fall. So gibt es unzählige Diskussionen zur Gegenüberstellung der beiden Scriptssprachen Perl 
und Python, die beide insbesondere auch zur maschinellen Verarbeitung von Sprache (Computerlinguistik, Korpuslinguistik etc.) verwendet werden. Natürlich gibt es deutliche technische Unterschiede zwischen den Sprachen. Die folgenden Zitate zeigen jedoch, dass auch immer „kulturelle“, „philosophische“ oder ideologische Gründe genannt (oder unterstellt) werden:

All languages have their points of strength and weakness except for Perl which is more of a religion than a programming language and has not faults whatsoever :-)

[...]

Perl's major disadvantages are:

1 It is not a trendy newkid on the block.

(http://www.perlmonks.org/?node_id=102231, 22.9.2020)

Perl's like vi - you pay up-front, with its golf syntax and scalar vs list DWIM (pronounced Dimwit I Am). It's unix shell on drugs.

(http://xahlee.info/comp/computer_languages_comparison_perl_python_ruby_javascript _php_lisp.html, 22.9.2020)

Perl is better.

Perl has almost no constraints. It's philosophy is that there is more than one way to do it (TIMTOWTDI, pronounced Tim Toady).

Python artificially restricts what you can do as a programmer. It's philosophy is that there should be one way to do it. If you don't agree with Guido's way of doing it, you're sh^t out of luck.

Basically, Python is Perl with training wheels. Training wheels are a great thing for a beginner, but eventually you should outgrow them. Yes, riding without training wheels is less safe. You can wreck and make a bloody mess of yourself. But you can also do things that you can't do if you have training wheels. You can go faster and do interesting and useful tricks that aren't possible otherwise. Perl gives you great power, but with great power comes great responsibility.

A big thing that Pythonistas tout as their superiority is that Python forces you to write clean code. That's true, it does... at the point of a gun, sometimes at the detriment of simplicity or brevity. Perl merely gives you the tools to write clean code (perltidy, perlcritic, use strict, / $\mathrm{x}$ option for commenting regexes) and gently encourages you to use them. (https:// www.quora.com/Which-is-better-Perl-or-Python-Which-one-is-more-robust-How-dothey-compare-with-each-other, 22.9.2020)

Einerseits werden Diskussionen um Computer von Topoi wie Utilitarismus und Purismus beherrscht, andererseits hat Programmcode immer eine Bedeutung und ist in kulturelle Praktiken eingebettet. Einer Programmiersprache werden Absichten unterstellt (sauber oder fantasievoll zu arbeiten) und die damit entstandenen Programme werden als Teil einer Kultur gelesen, die eine bestimmte Philosophie widerspiegeln. 
Kann man sich nun bei der Verwendung von Software oder von Programmiersprachen zur Erstellung von Visualisierungen diesen kulturellen Prägungen, diesen Coding Cultures einfach entziehen, indem man sich auf den Standpunkt stellt, nur Anwenderin oder Anwender zu sein? Im nächsten Abschnitt möchte ich konkret zeigen, welche Auswirkungen Coding Cultures bestimmter Technologien, die wichtig für die Erstellung von Visualisierungen sind, haben.

\subsubsection{Excel, R, Javascript, Perl, Python}

Anhand von vier Programmiersprachen und einer Software-Anwendung, die alle bei der computertechnischen Bearbeitung und Visualisierung von sprachlichen Daten eine bedeutende Rolle spielen, möchte ich die Auswirkungen von Coding Cultures auf die Visualisierungen selbst konkretisieren. Zunächst ein paar wenige Anmerkungen zu diesen Programmiersprachen bzw. zur Anwendung:

Excel von Microsoft ist eine bekannte proprietäre und kommerzielle Tabellenkalkulationssoftware, mit der Daten erfasst, manipuliert aber auch visualisiert werden können. Die Software gibt es seit 1985, zunächst für das Macintosh-Betriebssystem, und war innovativ, da es ein grafisches Benutzerinterface nutzte. Aus diagrammatischer Sicht bedeutet dies also, dass Excel als eine der ersten Softwares die Grundfigur der Tabelle als direkt mit einer Maus manipulierbare Tabelle anzeigte. ${ }^{24}$ Neben dem kommerziellen Excel, das ein Teil der Office-Suite von Microsoft ist, existiert mit „Calc“ als Teil von „LibreOffice“ oder „OpenOffice“25 eine Open-Source-Alternative mit ähnlichem Funktionsumfang.

R ist eine freie Programmiersprache, die insbesondere für statistische Berechnungen (deskriptive und explorative Statistik) und die Erstellung von Diagrammen designt wurde. ${ }^{26} \mathrm{R}$ existiert seit 1992 und wird laufend weiterentwickelt. Wie Javascript, Perl und Python muss auch R nicht kompiliert werden, sondern jeder Befehl wird direkt ausgeführt. R wird grundsätzlich direkt in einer Konsole ausgeführt, wobei es verschiedene Oberflächen wie RStudio ${ }^{27}$ gibt, die die Verwendung von R erleichtern.

Javascript ist eine Scriptsprache, die hauptsächlich für Webanwendungen entwickelt worden ist und 1995 von Netscape, dem Unternehmen, das den damals populärsten Webbrowser herstellte, in deren Browser eingebaut worden

24 VisiCalc von 1978 gilt als erstes Tabellenkalkulationsprogramm.

25 Vgl. https://de.libreoffice.org/ und https://www.openoffice.org/de/ (22. 9. 2020).

26 Vgl. https://www.r-project.org/ (22. 9. 2020).

27 Vgl. https://www.rstudio.com/ (22.9.2020). 
ist. Damit konnten beispielsweise Formulardaten auf Webseiten vor dem Absenden überprüft oder Text und Grafik manipuliert werden. Javascript-Code wird auf Seiten des Clients, also des die Webseite aufrufenden Browsers, in einer sogenannten „Sandbox“ ausgeführt, über deren Grenzen hinaus keine Zugriffe etwa auf das Dateisystem des Clients möglich sind.

Perl ist eine von Larry Wall entwickelte Scriptsprache, die 1987 veröffentlicht wurde und zunächst die Aufgabe hatte, Informationen aus verstreuten LogDateien auszulesen. Es ging also darum, Textdateien nach bestimmten Mustern zu durchsuchen. Wall, der Linguistik studiert hatte, orientierte sich bei der Entwicklung von Perl an der menschlichen Sprache, indem z. B. Befehle kontextsensitiv interpretiert werden und es meist verschiedene Ausdrucksmöglichkeiten gibt, die zum gleichen Ziel führen. Im Lauf der Zeit entwickelte sich Perl zu einer umfassenden Programmiersprache, die vielfältig eingesetzt wird. Lange war Perl häufig eingesetzte Sprache, um Text zu verarbeiten, so etwa in der Computerlinguistik. $^{28}$

Python ist ebenfalls eine Scriptsprache, die von Guido van Rossum erfunden worden ist und 1991 erstmals publiziert wurde. Ziel war größte Einfachheit und Übersichtlichkeit zu erreichen und eine umfangreiche Standardbibliothek von häufig benutzten Funktionen anzubieten. Übersichtlichkeit wird etwa dadurch erzwungen, dass korrekte Einrückungen der Codezeilen zwingend sind (was bei Perl und vielen anderen Sprachen nicht der Fall ist, wo Blöcke mit geschweiften Klammern o. ä. markiert werden), was automatisch zu einer gewissen Übersichtlichkeit führt. Gerade in textverarbeitenden Domänen wie der Computerlinguistik hat Python inzwischen Perl als wichtigste Programmiersprache abgelöst. ${ }^{29}$

Die erwähnten Technologien unterscheiden sich primär natürlich dadurch, dass Excel eine Software ist, bei der Programmierung nicht im Vordergrund steht (aber in Form von einbettbaren Scripts natürlich möglich ist), bei den anderen es sich jedoch um Programmiersprachen handelt, wo erst ein Script geschrieben werden muss, bevor eine Aufgabe gelöst werden kann. Allerdings ist der Unterschied kleiner als er auf den ersten Blick erscheint, da eine Sprache wie $\mathrm{R}$ beispielsweise oft innerhalb einer Umgebung wie Rstudio benutzt wird, die ein grafisches Benutzerinterface anbietet, um etwa Daten durch Menübefehle laden

28 Vgl. die Erhebung von Tiobe zur Verwendung von Perl: https://www.tiobe.com/tiobe-index/ perl/ (letzter Zugriff: 22. 9.2020).

29 Vgl. die Erhebung von Tiobe zur Verwendung von Python: https://www.tiobe.com/tiobe-index/python/ (letzter Zugriff: 22. 9. 2020); ferner ist zu beobachten, dass an vielen Instituten für Computerlinguistik inzwischen Python gelehrt wird. 
oder Datensätze grafisch dargestellt betrachten zu können. Bei den Programmiersprachen ist es jedoch so, dass jeder Befehl als Textanweisung einsehbar ist.

Zudem ist für Python, Perl, R und Javascript der Scripting-Ansatz typisch: Traditionell unterscheidet man zwischen Programmiersprachen, die kompiliert werden müssen, bevor sie ausgeführt werden können. Damit ist gemeint, dass das Programm in Maschinencode transformiert wird, der vom Computer verstanden wird. Dabei entsteht die ausführbare Datei, das Programm, dessen Quellcode nicht mehr eingesehen werden kann. Bei sogenannten Scriptsprachen werden Befehle einem Interpreter übergeben, der zum Zeitpunkt der Ausführung den Code interpretiert und in Maschinencode transformiert. Damit kann das Script, oder auch Teile davon, sofort ausgeführt werden. Zudem ist der Quellcode einsehbar: So kann jede/r Besucher/in einer Website den Javascript-Code, der die Funktionen beeinflusst, einsehen. Oder die R-Befehle, die zur Erstellung eines Diagramms verwendet worden sind, können veröffentlicht und von anderen selbst angewandt werden.

Scripting-Sprachen entsprechen damit deutlich den Hacker-Prinzipien von Open Source, Transparenz und Weitergabe des Quellcodes zur weiteren Veränderung, denn es ist naheliegend (oder im Fall von Javascript gar zwingend), den Quellcode bei den selbst erstellten Anwendungen offenzulegen. Somit kann er auch von anderen kopiert und für eigene Zwecke angepasst werden.

Excel entspricht diesen Prinzipien natürlich nicht. Microsoft lebt davon, dass die Software gekauft werden muss, nicht verändert und möglichst nicht weitergegeben werden kann. Bereits 1976 beklagte sich Bill Gates, der Gründer von Microsoft, in einem offenen Brief an die „Computer-Hobbyists“ über „Softwarediebstahl“ durch die zahlreichen Hobby-Computernutzer, die sein Altair BASIC nicht gekauft, sondern voneinander kopiert hätten. ${ }^{30}$

$\mathrm{R}$ ist bezüglich der Hacking-Philosophie ein Gegenentwurf zu kommerziellen Softwarepaketen wie SPSS oder SAS, die proprietären Softwarelösungen für ähnliche Zwecke. Der Quellcode dieser Programme ist nicht offen und die Weiterentwicklung der Software ist in fester Hand der Firmen IBM bzw. SAS Institute. Über das Basismodul hinausgehende Funktionen müssen zusätzlich gekauft werden. Bei R hingegen gibt es eine Gemeinde von Nutzerinnen und Nutzern, die R weiterentwickelt und um Module mit speziellen Funktionen erweitert.

Neben den lizenzrechtlichen Aspekten von Open und Closed Source, proprietärer und freier Software, merkt man den unterschiedlichen Technologien aber ihre Einbettung in unterschiedliche Coding Cultures an. Ein Beispiel betrifft Javascript: Basierend auf Javascript gibt es für den Bereich der Datenvisualisie-

30 Vgl. https://en.wikipedia.org/wiki/Open_Letter_to_Hobbyists (22.9. 2020). 
rung einige Bibliotheken, darunter z. B. „D3“ (Bostock et al. 2011), die auch für die in den Kapiteln 6 und 7 gezeigten Visualisierungen verwendet werden. Ein anderes Beispiel ist die Bibliothek „P5“31, die auf der Programmierumgebung „Processing“ (Fry 2008) beruht und insbesondere in Design- und Digitalkunstkreisen beliebt ist. Ein Werbevideo für P5 mit der Hauptentwicklerin Lauren McCarthy und dem Mitentwickler Dan Shiffman ist symptomatisch für eine bestimmte Coding Culture des ,just do it“, wie ich es nennen möchte. ${ }^{32}$ Nach einer Begrüßung durch McCarthy zeigt Shiffman ein paar P5-Befehle, um interaktive Grafiken zu zeichnen. Das Video selbst ist jedoch ebenfalls mit P5 programmiert, so dass der Betrachter oder die Betrachterin mit den von Shiffman vorgestellten grafischen Elemente (mit den dafür nötigen Codezeilen) direkt interagieren kann. Die Demo betont die Einfachheit, mit der programmiert werden kann. Die zu Beginn genannten Eigenschaften von P5 sind „Programming for the Arts, Made for Teaching, Extensible with Add-Ons, Free to Download, Open Source“. Auch am Ende der Demo benennt McCarthy die Zielgruppe von P5, die deutlich macht, dass nicht (nur) professionelle Programmierer/innen angesprochen werden, sondern auch Programmier-Laien.
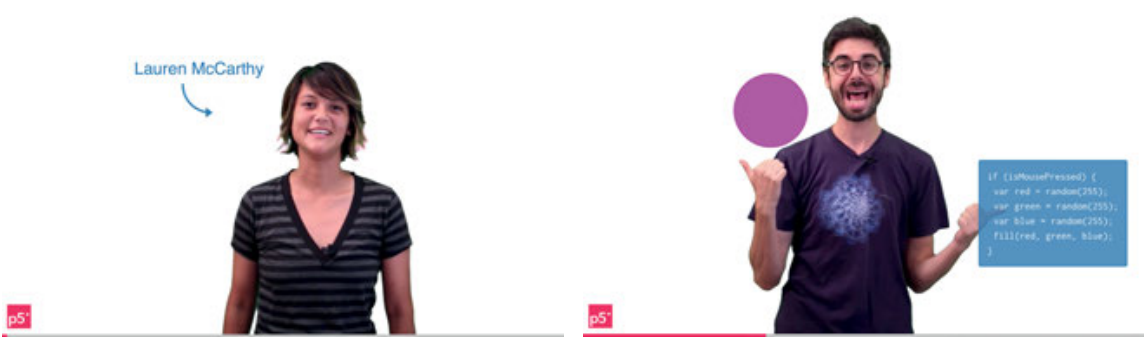

Abb. 34: Demo der Javascript-Bibliothek P5 durch Lauren McCarthy und Ben Shiffman (http:// hello.p5js.org, 22.9. 2020)

Typisch für Scriptsprachen sind auch umfangreiche Sammlungen von Codeschnipseln und Beispielanwendungen, aus denen man sich den Code für die eigene Anwendung zusammenstellen kann. Für D3 etwa, die bereits oben erwähnte Javascript-Bibliothek für Datenvisualisierungen, ist auf der Website der

31 Vgl. https://p5js.org/ (22.9.2020).

32 Das Video ist hier einsehbar: http://hello.p5js.org (22.9. 2020). 
Bibliothek eine „Gallery“ mit „Visual Index“ von unterschiedlichen Visualisierungen verfügbar, auf denen man eine eigene Anwendung aufbauen kann. ${ }^{33}$

Die Javascript-Bibliotheken, aber auch R, Perl und Python sind Beispiele für eine neue Kultur der Software-Entwicklung, die ich wie folgt charakterisieren würde:

- Präferiert wird Open-Source-Software - der Quellcode liegt offen, d. h. frei zugänglich, da und kann einfach weiterverwendet und für neue Applikationen angepasst werden.

- Entprofessionalisierung: Nicht nur Informatiker/innen können ansprechende Anwendungen bauen, sondern auch informatiktechnische Laien.

- Experimentierfreudigkeit: Software wird nicht nur nach strikten Bauplänen systematisch entwickelt, sondern experimentierend und im positiven Sinn eklektisch und erratisch gebaut.

- Selbstermächtigung: Die grafischen Benutzeroberflächen vereinfachten zwar die Benutzung der Computer, nahmen den Nutzer/innen aber auch immer mehr Einflussmöglichkeiten auf das System (vgl. dazu Pratschke 2008). Die aktuellen Smartphones und Tablet-Computer in Verbindung mit App-Stores sind ein gutes Beispiel: Auf ihnen soll konsumiert und nicht Neues geschaffen werden, schon gar nicht unter Umgehung der Oberfläche. Durch die Aspekte Open-Source, Entprofessionalisierung und Experimentierfreudigkeit ist es jedoch wieder einfacher geworden, sich gegenüber dem Computer (und den Computerkonzernen) selbst zu ,ermächtigen', also zu programmieren.

Dies sind Aspekte, für die die Hacking-Kultur schon immer stand und im Zuge der breiten Digitalisierung auch in den Geisteswissenschaften und nach einer starken Phase der kommerziellen Entwicklung von proprietärer Software nun wieder in Mode sind.

Allerdings: Trotz Experimentierfreudigkeit und Selbstermächtigung führen die genannten Technologien auch wiederum zu Kanonisierungsphänomenen, was für meinen Untersuchungsgegenstand von besonderem Interesse ist. D3 etwa ist so erfolgreich und verbreitet, dass diese Bibliothek das Aussehen und die Funktionsweisen vieler Datenvisualisierungen bestimmt, da es eben besonders einfach ist, bestehenden Code $\mathrm{zu}$ verwenden und an die eigenen Daten anzupassen. Es ist immer schneller, dem Kanon entsprechende Visualisierungen zu erstellen, als gänzlich neue Wege zu gehen.

Mit der Wahl bestimmter technologischer Mittel zur Erstellung von Visualisierungen wird man Teil einer bestimmten Coding Culture. Es gibt die Wahl-

33 Vgl. https://github.com/d3/d3/wiki/Gallery (22.9.2020). 
möglichkeit, sich zu einer bestimmten Coding Culture zu positionieren und sich damit auch einer bestimmten Wissenschaftskultur anzuschließen. Das beginnt schon damit, dass man sich gegen Ideale der Entprofessionalisierung und Selbstermächtigung stellen kann, indem man die Visualisierung jemanden übergibt, die/der sich professionell damit beschäftigt. Die Visual Analytics als Disziplin sind daran interessiert, sich als professionelle Datenvisualisierer/innen und damit als Denkkollektiv zu etablieren, indem sie Standards für Visualisierungen definieren und Hürden für Außenstehende aufbauen. Ein Beispiel dafür sind die Durchführung von wissenschaftlichen Kongressen zu Visualisierungsproblemen, bei denen mit dem Reviewverfahren ein Mittel zur Verfügung steht, den Zugang zum Denkkollektiv zu regulieren.

Alternativ kann man sich auf den Standpunkt stellen, die Visualisierung von Daten als integralen Teil der gesamten Datenaufbereitung, -Analyse und Interpretation zu sehen und deswegen eine nicht-professionelle Visualisierung in den Händen des eigenen disziplinären Teams vorzunehmen. Je nach Entscheidung werden damit auch andere technologische Werkzeuge ausgewählt werden. 